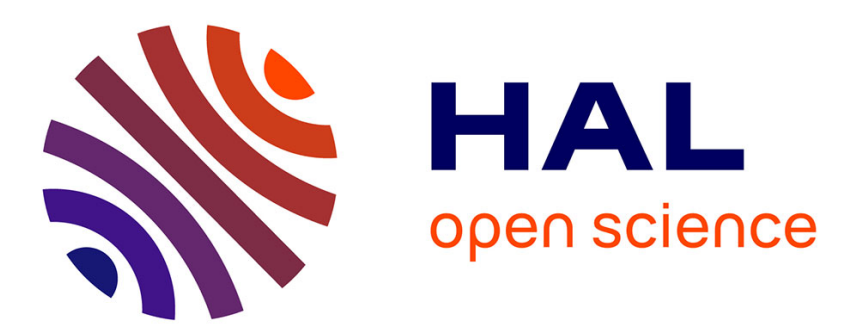

\title{
Probabilistic Safety Analysis of the Collision Between a Space Debris and a Satellite with an Island Particle Algorithm
}

Christelle Vergé, Jérôme Morio, Pierre del Moral, Juan Carlos Dolado Pérez

\section{- To cite this version:}

Christelle Vergé, Jérôme Morio, Pierre del Moral, Juan Carlos Dolado Pérez. Probabilistic Safety Analysis of the Collision Between a Space Debris and a Satellite with an Island Particle Algorithm. Springer Optimization and Its Applications, 2017, 114, pp.443-457. 10.1007/978-3-319-41508-6_17. hal-01436202

\section{HAL Id: hal-01436202 https://hal.science/hal-01436202}

Submitted on 16 Jan 2017

HAL is a multi-disciplinary open access archive for the deposit and dissemination of scientific research documents, whether they are published or not. The documents may come from teaching and research institutions in France or abroad, or from public or private research centers.
L'archive ouverte pluridisciplinaire HAL, est destinée au dépôt et à la diffusion de documents scientifiques de niveau recherche, publiés ou non, émanant des établissements d'enseignement et de recherche français ou étrangers, des laboratoires publics ou privés. 


\title{
Probabilistic safety analysis of the collision between a space debris and a satellite with an island particle algorithm
}

\author{
Christelle Vergé ${ }^{1,2,3}$ Jérôme Morio ${ }^{4, *}$ Pierre Del Moral ${ }^{5}$ \\ Juan Carlos Dolado Pérez ${ }^{2}$
}

\begin{abstract}
Collision between satellites and space debris seldom happens, but the loss of a satellite by collision may have catastrophic consequences both for the satellite mission and for the space environment. To support the decision to trigger off a collision avoidance manoeuver, an adapted tool is the determination of the collision probability between debris and satellite. This probability estimation can be performed with rare event simulation techniques when Monte Carlo techniques are not enough accurate. In this chapter, we focus on analyzing the influence of different simulation parameters (such as the drag coefficient) that are set for to simplify the simulation, on the collision probability estimation. A bad estimation of these simulation parameters can strongly modify rare event probability estimations. We design here a new island particle Markov chain Monte Carlo algorithm to determine the parameters that, in case of bad estimation, tend to increase the collision probability value. This algorithm also gives an estimate of the collision probability maximum taking into account the likelihood of the parameters. The principles of this statistical technique are described throughout this chapter.
\end{abstract}

Key words: Rare event, sequential Monte Carlo, island particle models, debris, satellite, collision, adaptive splitting technique

\footnotetext{
* corresponding author

Email address: jerome.morio@onera.fr (Jérôme Morio).

1 Onera - The French Aerospace Lab, F-91761 Palaiseau

2 CNES, 18 avenue Edouard Belin, 31401 Toulouse

3 Centre de Mathématiques Appliquées, Route de Saclay, 91128 Palaiseau

4 DCPS/UFTMIP/ONERA, Onera - The French Aerospace Lab, F-31055 Toulouse

5 School of Mathematics and Statistics, University of New South Wales, High Street, Kensington, Sydney NSW 2052
} 


\section{Introduction}

On February $10^{\text {th }} 2009$, active commercial satellite Iridium-33 and out of order Russian satellite Cosmos-2251 collided [Kelso, 2009]. The impact produced more than 2000 trackable debris. Most of them may destroy any satellite, whether in use or not, they might encounter. The safest practice for satellites that encounter space debris is to avoid collision. Avoidance maneuvers are an efficient mean to reduce the collision probability between two orbiting objects, nevertheless they consume fuel reducing the operational lifetime of the satellite and they perturb the operational mission of the satellite. Consequently, satellite safety responsible teams have to take into account the operational mission prior to the definition of a collision avoidance maneuver and try to combine, whenever possible, planned station keeping maneuvers with collision avoidance maneuvers. Avoidance maneuvers are decided, among other parameters, based on the estimated collision probability.

The orbital motion of the space objects is simulated using a simplified deterministic dynamical model that may be considered as an input-output function where the random inputs are, for instance, the position and the speed of the debris and of the satellite as well as other dynamic parameters as the drag coefficient, and the output is the minimum distance between the debris and the satellite. The collision probability is then estimated on this output. This input-output function can be seen as a "black-box" with random inputs. Some parameters, denoted by a vector $\Theta$, in black-box functions are implicitly set, such as parameters of the model (the drag coefficient for instance) or of the input parametric model density, and their value influences the collision probability estimation. These hypotheses are often assumed for simplification and computational reasons. From a risk analysis point of view, it is interesting to determine the variability of the collision probability w. r. t. the uncertainty on theses input parameters $\Theta$ or w. r. t. one particular parameter, and to quantify the impact of such tuning in the realization of a collision. Of course, different values of $\Theta$ can strongly modify rare event probability estimation and sometimes miss a risk situation. The issue of concern in safety would be to underestimate a risk because of a bad tuning of model parameters $\Theta$. That is why in this paper we propose to estimate the law of the parameters $\Theta$ conditionally on a collision between the debris and the satellite. We develop in this chapter the $\mathrm{SMC}^{2}$ (Sequential Monte Carlo Square) algorithm to estimate this kind of targeted laws introduced [Chopin et al., 2013] to do filtering on hidden Markov models. We apply this island particle algorithm to debris satellite collision use case and analyse its results for the system safety. 


\section{Debris satellite collision simulation}

We consider two space objects (a debris and a satellite) orbiting around an Earth centered inertial reference frame. Their geometry is assumed spherical (i.e. the objects have a high tumbling motion when compared with their orbital period) and we assume that we perfectly know the radius of such sphere and the mass of the objects. We wonder about the relative position of the two satellites and ask whether the distance between the two objects could be smaller than a conflict distance $T$ during the given time span $I$. To model the orbital motion of both space objects, we consider a general perturbation approach where the original equations of motion are replaced with an analytical approximation that captures the essential character of the motion over some limited time interval, which also enables analytical integration of the equations. SGP4 model [Miura, 2009] is used to propagate the trajectories of debris and satellite according to the time. At time $t$, the space objects will be represented by their 6 -dimensional state vectors $\vec{s}_{1}(t)$ and $\vec{s}_{2}(t)$, i.e. their 3 -dimensional position vectors $\vec{r}_{1}(t)$ and $\vec{r}_{2}(t)$ and their 3 -dimensional speed vectors $\vec{v}_{1}(t)$ and $\vec{v}_{2}(t)$ such that $\vec{s}_{i}=\left(\vec{r}_{i}, \vec{v}_{i}\right)$. The initial conditions in the proposed example is defined in terms of two line elements (TLE), similar to those provided by NORAD (North American Aerospace Defense Command), as the SGP4 model is used for the orbital propagation of the considered objects. The initial condition value is denoted $\vec{s}_{i}^{m}$ at a given time $t_{i}^{m}$. SGP4 enables us to propagate the orbit of both space objects through time, denoted by a scalar continuous function $\nu$ such that

$$
\begin{gathered}
\forall i \in\{1,2\}, \forall t \in I, \vec{s}_{i}(t)=\nu\left(\vec{s}_{i}^{m}, t_{i}^{m}, t\right), \\
\delta=\min _{t \in I}\left\{\left\|\vec{r}_{2}-\vec{r}_{1}\right\|(t)\right\} .
\end{gathered}
$$

The function of time $t \in I \mapsto\left\|\vec{r}_{2}-\vec{r}_{1}\right\|(t)$ makes $\delta$ available through numerical optimisation in a deterministic approach. In fact, the position and velocity of space objects are estimated from more or less imprecise measurements. While the measurement means used for satellites (e.g. GPS, laser) result in a reasonable orbital accuracy (e.g. several tens of meters) the measurement means used for debris and uncooperative space-objects (e.g. mainly radar and telescopes) could result in quite imprecise orbits (e.g. several hundred of meters or few kilometers). This lack of accuracy will depend on a great number of factors. TLEs sum up this information and feed the models with the couple $\left(\vec{s}_{i}^{m}, t_{i}^{m}\right)$ for $i=1,2$, but to cope with their uncertainty, we have added independent and identically distributed Gaussian noises to the model inputs $\vec{s}_{i}^{m}$.

Debris satellite conflict may be modelled as an input-output function where:

- the input $X$ represents the position and the speed of the debris space (the position and the speed of the satellite are assumed to be known). $X$ is a 6dimensional multivariate normal random vector of mean $\left(\Theta_{1}, \Theta_{2}, \Theta_{3}, \Theta_{4}, \Theta_{5}, \Theta_{6}\right)^{t}$ 
and covariance matrix is equal to the identity matrix defined on a measurable space $(\mathrm{X}, \mathcal{X})$. The means corresponds to the debris measurement errors on its position and speed;

- the input-output function $\phi$ enables to propagate the debris and satellite trajectories with the SGP4 model during $I$. The input-output code includes the transformation that allows to switch from the standard space of the input to the physical space in which evolve the satellite and debris position and speed. The function $\phi$ is a continuous positive scalar function $\phi: \mathbb{R}^{6} \rightarrow$ $\mathbb{R}$ and is static;

- the error on the drag coefficient which is considered inside the function $\phi$ is also random and follows a normal distribution with mean $\Theta_{7}$ and variance 1 ;

- the output $Y$ is the minimum distance between the debris and the satellite during $I$. We assume that it is a positive random variable.

The complete set of model parameters is summed up in the vector $\Theta=$ $\left(\Theta_{1}, \Theta_{2}, \Theta_{3}, \Theta_{4}, \Theta_{5}, \Theta_{6}, \Theta_{7}\right)^{t}$. The quantity of interest on the output $Y$ is the probability

$$
\mathbb{P}(Y<T)=\mathbb{P}(\phi(X)<T) .
$$

When the event $\{\phi(X)<T\}$ is rare relatively to the available simulation budget (which is often the case in safety and reliability issues), different algorithms described in [Sobol, 1994], [Bucklew, 2004], [Rubinstein and Kroese, 2004], [Zhang, 1996], [Bjerager, 1991], [Botev and Kroese, 2012], [Cérou et al., 2012] have notably been proposed to estimate accurately its probability.

\section{Basics of safety analysis}

In the present chapter, one focuses on the case where the law of $X$ is uncertain and depends upon unknown parameters. We assume that $X$ is distributed according to a well known parametric model and its parameters, denoted by a random vector $\Theta$, have a probability density $\nu$. We also suppose that $\Theta$ has a density $f_{\Theta}$ w. r. t. a dominating measure of reference $\lambda$, that is

$$
\nu(d \theta)=f_{\Theta}(\theta) \lambda(d \theta) .
$$

In the application considered here, $X$ is a random vector with a multivariate normal distribution, and $\Theta$ describe the mean of $X$. It corresponds notably to realistic applications where it is not always possible to evaluate accurately the density of input parameters. This formalism enables thus to consider a large range of input probability density function.

The probability of interest $\mathbb{P}(Y<T)$ depends of course on $\Theta$ and thus on the distribution $\nu$. In safety applications, it is important to estimate a superior 
bound of the rare event probability $\mathbb{P}(Y<T)$ taking also into account the prior on $\Theta$. The prior on $\Theta$ is important since unrealistic bad tuning values of $\Theta$ which lead to high probabilities $\mathbb{P}(Y<T)$ are not relevant. The idea of this chapter is thus to determine the distribution of $\Theta$ conditionally on the fact that $Y$ does not exceed the threshold $T$. This distribution, denoted by $\pi$, will be referred to in the sequel as the target law.

In the further development, when there is no confusion, we sometimes write $\mathbb{P}(Y<T \mid \theta)$ instead of $\mathbb{P}(Y<T \mid \Theta=\theta)$.

Note that using the Bayes' formula, the target law can be written

$$
\pi(d \theta)=\frac{1}{\mathbb{P}(Y<T)} \mathbb{P}(Y<T \mid \theta) \nu(d \theta)
$$

We propose in this paper a SMC (Sequential Monte Carlo) algorithm which evolves according to iterative selection and mutation steps, and which approximates $\pi$ when the number of particles gets large. This algorithm requires the estimation of $\mathbb{P}(\phi(X)<T \mid \Theta=\theta)$ for different settings of parameter $\theta$. For that purpose, we describe the splitting algorithm that enables us to estimate this probability with accuracy.

\section{The $\mathrm{SMC}^{2}$ algorithm}

\subsection{Principle}

The $\mathrm{SMC}^{2}$ algorithm is based on the use of two sets of particles to iteratively approach $\pi$. The first set of particles is defined on the parameter $\Theta$ and the second set of particles is useful to estimate the probabilities $\mathbb{P}(Y<T \mid \theta)$. The complete demonstration of interacting particles systems (IPS) convergence and the link with Feynman-Kac framework is given in Moral et al. [2012]. Define $T_{1}, T_{2}, \ldots, T_{n}=T$ a serie of decreasing thresholds and denote for all $i \in \llbracket 0, n \rrbracket$

$$
\pi_{i}=\frac{1}{\mathbb{P}\left(Y<T_{i}\right)} \mathbb{P}\left(Y<T_{i} \mid \theta\right) \nu(d \theta) .
$$

The target law is of course $\pi=\pi_{n}$. The probability law $\pi_{n}$ is thus proportional to

$$
\begin{gathered}
\pi_{n} \propto \mathbb{P}\left(Y<T_{n} \mid \theta\right) \nu(d \theta) \\
\pi_{n} \propto H_{n}(\theta) \nu(d \theta),
\end{gathered}
$$


where $H_{n}(\theta)=\mathbb{P}\left(Y<T_{n} \mid \theta\right)$. The term $H_{n}(\theta)$ can be expressed as a product of conditional probabilities

$$
H_{n}(\theta)=\left[\prod_{p=1}^{n-1} \mathbb{P}\left(Y<T_{p+1} \mid Y<T_{p}, \theta\right)\right] \times \mathbb{P}\left(Y<T_{1} \mid \theta\right)=\prod_{p=0}^{n-1} h_{p}(\theta)
$$

with

$$
\left\{\begin{array}{l}
h_{p}(\theta)=\mathbb{P}\left(Y<T_{p+1} \mid Y<T_{p}, \theta\right) \\
h_{0}(\theta)=\mathbb{P}\left(Y<T_{1} \mid \theta\right)
\end{array}\right.
$$

In this notation, we have

$$
\pi_{n} \propto \prod_{p=0}^{n-1} h_{p}(\theta) \nu(d \theta)
$$

One can also remark that $H_{p}=H_{p-1} \times h_{p-1}$ and consequently the link between $\pi_{p+1}$ and $\pi_{p}$ can be written on the following way

$$
\pi_{p+1}=\psi_{h_{p}}\left(\pi_{p}\right)
$$

where $\psi_{h_{p}}$ is the so-called Boltzmann-Gibbs transformation. Let $\mathcal{P}(\mathbb{E})$ be the set of probability measures on $\mathbb{E}$. For all positive bounded function $G$, the Boltzmann-Gibbs transformation $\Psi_{G}: \mathcal{P}(\mathbb{E}) \rightarrow \mathcal{P}(\mathbb{E})$ is defined for all $\mu \in$ $\mathcal{P}(\mathbb{E})$ such that $\mu(G)=\int G(x) \mu(d x)>0$ by

$$
\Psi_{G}(\mu)(d x):=\frac{1}{\mu(G)} G(x) \mu(d x) .
$$

If one assumes that it is possible to determine a Markovian kernel $M_{p}$ that let $\pi_{p}$ invariant (which is not restrictive using, for example, a stage of the acceptance/rejection of Metropolis-Hastings algorithm) we have

$$
\pi_{p}=\left(\pi_{p} M_{p}\right)(d \theta)=\int \pi_{p}\left(d \theta^{\prime}\right) M_{p}\left(\theta^{\prime}, d \theta\right)
$$

This yields the evolution equation

$$
\pi_{p+1}=\psi_{h_{p}}\left(\pi_{p}\right) M_{p+1}
$$

Equation 6 may be cast in the Feynman-Kac framework and then, each measure $\pi_{p}$ can be approximated by an IPS which evolves with selection steps related to the so called potential functions $h_{p}$ and mutation steps related to 
the Markov kernel $M_{p}$. Denote by $\left\{\left(\theta_{p}^{1}, \ldots, \theta_{p}^{N_{1}}\right)\right\}_{n \geq 0}$ a system of $N_{1}$ particles.

$$
\left[\begin{array}{c}
\theta_{0}^{1} \\
\cdot \\
\cdot \\
\cdot \\
\cdot \\
\theta_{0}^{N_{1}}
\end{array}\right] \stackrel{\widehat{\theta}_{0}^{1}}{\stackrel{\text { Selection }}{\longrightarrow}}\left[\begin{array}{c}
\theta_{1}^{1} \\
\cdot \\
\cdot \\
\cdot \\
\cdot \\
\widehat{\theta}_{0}^{N_{1}}
\end{array}\right] \stackrel{\text { Mutation }}{\longrightarrow}\left[\begin{array}{c}
\widehat{\theta}_{1}^{1} \\
\cdot \\
\cdot \\
\cdot \\
\cdot \\
\theta_{1}^{N_{1}}
\end{array}\right] \stackrel{\text { Melection }}{\longrightarrow}\left[\begin{array}{c}
\cdot \\
\cdot \\
\cdot \\
\widehat{\theta}_{1}^{N_{1}}
\end{array}\right]
$$

The selection stage consists in sampling $\left\{\widehat{\theta}_{p}^{i}\right\}_{i=1}^{N_{1}}$ independently according to the probability measure $\psi_{h_{p}}$, i.e. selecting the particles $\left\{\theta_{p}^{i}\right\}_{i=1}^{N_{1}}$ with probabilities proportional to their weights $\left\{h_{p}\left(\theta_{p}^{i}\right)\right\}_{i=1}^{N_{1}}$. The mutation stage consists in updating the selected particles conditionally independently using the Markov kernel $M_{p+1}$ that let $\pi_{p+1}$ invariant. This step enables to increase the diversity of $\widehat{\theta}_{p}$ without changing its probability law, that is already close to $\pi_{p+1}$. The Feynman-Kac theory Moral et al. [2012] ensures that at each transition stage $p:$

$$
\frac{1}{N_{1}} \sum_{i=1}^{N_{1}} \delta_{\theta_{p}^{i}} \underset{N_{1} \rightarrow+\infty}{\longrightarrow} \pi_{p} .
$$

Thus, at the end of the $n^{\text {th }}$ transition stage, the system of particles converges to the target law $\pi_{n}$ so that

$$
\frac{1}{N_{1}} \sum_{i=1}^{N_{1}} \delta_{\theta_{n}^{i}} \underset{N_{1} \rightarrow+\infty}{\longrightarrow} \pi_{n} .
$$

Nevertheless, the knowledge of $h_{p}$ is required to apply the different selection/mutation stages. In practice, $h_{p}\left(\theta_{p}^{i}\right)$ is not analytically computable but can be estimated by defining a new set of particles $\left\{\xi_{p}^{i, j}\right\}_{j=1}^{N_{2}}$ on the random variable $X$ conditionally to the different thresholds $T_{p}$ and associated to each $\theta_{p}^{i}$

\subsection{Description}

Consider $\left\{\theta_{0}^{i}\right\}_{i=1}^{N_{1}}$ generated with probability law $\nu$. At iteration $k$ of the algorithm, with $k \geq 1$, we assume that particles $\left\{\theta_{k}^{i}\right\}_{i=1}^{N_{1}}$ are available and then, the interacting island algorithm consists in two iterative type stages: 
- Selection stage The selection stage consists in choosing randomly and independently $N_{1}$ particles amongst $\left\{\theta_{k}^{i}\right\}_{i=1}^{N_{1}}$ with probabilities proportional to their weights $\left\{h_{k}\left(\theta_{k}^{i}\right)\right\}_{i=1}^{N_{1}}$. Thus, the particles with low weights are killed whereas those with high weights are multiplied. The number of particles is kept constant in this stage and a new set particles $\left\{\widehat{\theta}_{k}^{i}\right\}_{i=1}^{N_{1}}$ can be defined. Remind that the potential functions $h_{k}$ are defined by:

$$
\left\{\begin{array}{l}
h_{k}\left(\theta_{k}^{i}\right)=\mathbb{P}\left(Y<T_{k+1} \mid Y<T_{k}, \theta=\theta_{k}^{i}\right), k \geq 1 \\
h_{0}\left(\theta_{0}^{i}\right)=\mathbb{P}\left(X<T_{1} \mid \theta=\theta_{0}^{i}\right)
\end{array}\right.
$$

These quantities have to be computed.

- Mutation stage Even if the number of particles is still equal to $N_{1}$, some particles have been duplicated, so we apply a Markov kernel to increase the diversity of the particles. Building a $\pi_{k+1}$-reversible transition kernel that let $\pi_{k+1}$ invariant is the objective of mutation stage. For that purpose, the acceptance/rejection step of the Metropolis-Hastings algorithm Tierney [1994] is useful. This approach results in the exploration of $\Theta$ space set without changing the $\left(\widehat{\theta}_{k}^{i}\right)_{1 \leq i \leq N_{1}}$ distribution and the increase of the particle diversity. A new particle $\theta_{k}^{i \prime}$ is proposed with a $\nu$-reversible kernel $Q$. The acceptation rate of a new proposal is consequently $1 \wedge \frac{H_{k+1}\left(\widehat{\theta}_{k}^{\prime \prime}\right)}{H_{k+1}\left(\widehat{\theta}_{k}^{i}\right)}$. If $H_{k+1}\left(\widehat{\theta}_{k}^{i \prime}\right)>H_{k+1}\left(\widehat{\theta}_{k}^{i}\right)$, the proposal $\widehat{\theta}_{k}^{i \prime}$ is automatically accepted and replaces $\hat{\theta}_{k}^{i}$ in the set of current particles. Otherwise, the proposal $\widehat{\theta}_{k}^{i \prime}$ is accepted with probability $\frac{H_{k+1}\left(\widehat{\theta}_{k}^{\prime \prime}\right)}{H_{k+1}\left(\widehat{\theta}_{k}^{i}\right)}$. This acceptance/rejection procedure is repeated $N_{a p p}$ times to decrease the correlation between the particles. At the end of this stage, a new set of particles $\left\{\theta_{k+1}^{i}\right\}_{1=i}^{N_{1}}$ can be defined.

Mutation and selection stage are applied $n$ times until reaching the target threshold $T_{n}$. At the end of the algorithm, the particles $\left\{\theta_{n}^{i}\right\}_{1=i}^{N_{1}}$ provides an estimate of $\pi_{n}$ :

$$
\widehat{\pi}_{n}^{N_{1}}=\frac{1}{N_{1}} \sum_{i=1}^{N_{1}} \delta_{\theta_{n}^{i}}
$$

For $i \in\left[1, N_{1}\right]$ and $k \in[0, n]$, the point is to estimate each probability $\left\{h_{l}\left(\theta_{k}^{i}\right)\right\}_{1 \leq l \leq k}$. It can be done with another interacting particle system (also called, in that case, sequential Monte Carlo, importance splitting, subset simulation or subset sampling). It is a rare event estimation technique which considers the estimation of several conditional probabilities that are easier to evaluate than estimate only one probability through a very tough simulation. Its principle is also based on selection and mutation stages. Let us define $\left\{\xi_{0}^{i, j}\right\}_{j=1}^{N_{2}}$ with probability density $f_{X \mid \theta_{j}^{i}}$ w.r.t. $\lambda_{X}$, i.e. the density of $X$ knowing $\Theta=\theta_{k}^{i}$. At iteration $l$ of the algorithm, we assume that particles $\left\{\xi_{l}^{i, j}\right\}_{j=1}^{N_{2}}$ are available and then IPS consists in two iterative stages: 
- Selection stage The selection stage consists in choosing randomly and independently $N_{2}$ particles amongst the particles $\left\{\xi_{l}^{i, j}\right\}_{j=1}^{N_{2}}$ which are above $T_{l}$. The particles which have not reached the threshold $T_{l}$ are thus killed. The number of particles is kept constant, and a new set of particles $\left\{\widehat{\xi}_{l}^{i, j}\right\}_{j=1}^{N_{2}}$ can be defined.

- Mutation stage The mutation stage is patterned with acceptance/rejection principle using the Metropolis-Hastings algorithm Tierney [1994]. A new particle $\widehat{\xi}_{l}^{i, j \prime}$ is then proposed with a Markov kernel $\tilde{Q}$. If $\phi\left(\widehat{\xi}_{l}^{i, j \prime}\right)<T_{l}$, then the proposal is accepted with probability $1 \wedge \frac{\left.f_{X \mid \theta_{l}^{i}} \widehat{\xi}_{l}^{i, j}\right) \tilde{Q}\left(\widetilde{\xi}_{l}^{,, j}, \widehat{\xi}_{l}^{i, j}\right)}{\left.f_{X \mid \theta_{l}^{i}} \widehat{\xi}_{l}^{i, j}\right) \tilde{Q}\left(\widehat{\xi}_{l}^{i, j}, \widehat{\xi}_{l}^{i, j \prime}\right)}$ and $\widehat{\xi}_{l}^{i, j \prime}$ replaces $\widehat{\xi}_{l}^{i, j}$ in the set of current particles. If $\phi\left(\widehat{\xi}_{l}^{i, j \prime}\right)>T_{l}$, the proposal is automatically rejected and the particle $\widehat{\xi}_{l}^{i, j}$ is remained. This acceptance/rejection procedure is repeated $N_{a p p 2}$ times to decrease the correlation between the particles. At the end of this stage, a new set of particles $\left\{\xi_{l+1}^{i, j}\right\}_{j=1}^{N_{2}}$ can be defined. An estimate $\widehat{h}_{l}\left(\theta_{k}^{i}\right)$ of $h_{l}\left(\theta_{k}^{i}\right)=\mathbb{P}\left(Y<T_{l+1} \mid Y<\right.$ $\left.T_{l}, \Theta=\theta_{k}^{i}\right)$ is given by the ratio between the number of $\left\{\xi_{l}^{i, j}\right\}_{j=1}^{N_{2}}$ particles such that $\phi\left(\xi_{l}^{i, j}\right)<T_{l+1}$ and the total number of particles $N_{2}$.

Mutation and selection stages are applied $k$ times until reaching the target threshold $T_{k}$. At the end of the algorithm, $H_{k+1}\left(\theta_{k}^{i}\right)=\mathbb{P}\left(Y<T_{k+1} \mid \theta_{k}^{i}\right)$ is estimated by

$$
\widehat{H}_{k+1}\left(\theta_{k}^{i}\right)=\prod_{l=0}^{k} \hat{h}_{l}
$$

For a given particle $\theta_{k}^{i}$, a complete set of particles $\left\{\xi_{l}^{i, j}\right\}_{\substack{1 \leq l \leq k \\ 1 \leq j \leq N_{2}}}$ is thus generated. An island particle is thus constituted of a particle $\theta_{k}^{i}$ and its associated $\left\{\xi_{l}^{i, j}\right\}_{1 \leq l \leq k}^{1 \leq j \leq N_{2}}$ particle set.

The $\mathrm{SMC}^{2}$ algorithm is described more precisely in Algorithm 1. Interacting particle system for probability estimation required in Algorithm 1 is developed in Algorithm 2.

The determination of $Q$ and $\tilde{Q}$, in the general case, implies the use of MetropolisHastings algorithm. Nevertheless, if $\mu$ is a standard normal distribution, a transition from $x$ to $z$ defined with the following expression

$$
x \mapsto z=\sqrt{1-a} x+\sqrt{a} W
$$

where $W \sim \mathcal{N}(0,1)$ and $a$ is scalar parameter such as $a \in[0,1]$, is $\mu$-reversible. In order to use equation 7 instead of Metropolis-Hastings algorithm, it is also possible to apply a transformation on the variables $X$ or $\Theta$ so that they follow a standard normal PDF. Depending on the available information on the PDF of $X$, several transformations can be proposed Nataf [1962], Pei-Ling and Kiureghian [1991], Lebrun and Dutfoy [2009b], Rosenblatt [1952] and Lebrun and Dutfoy [2009a]. 


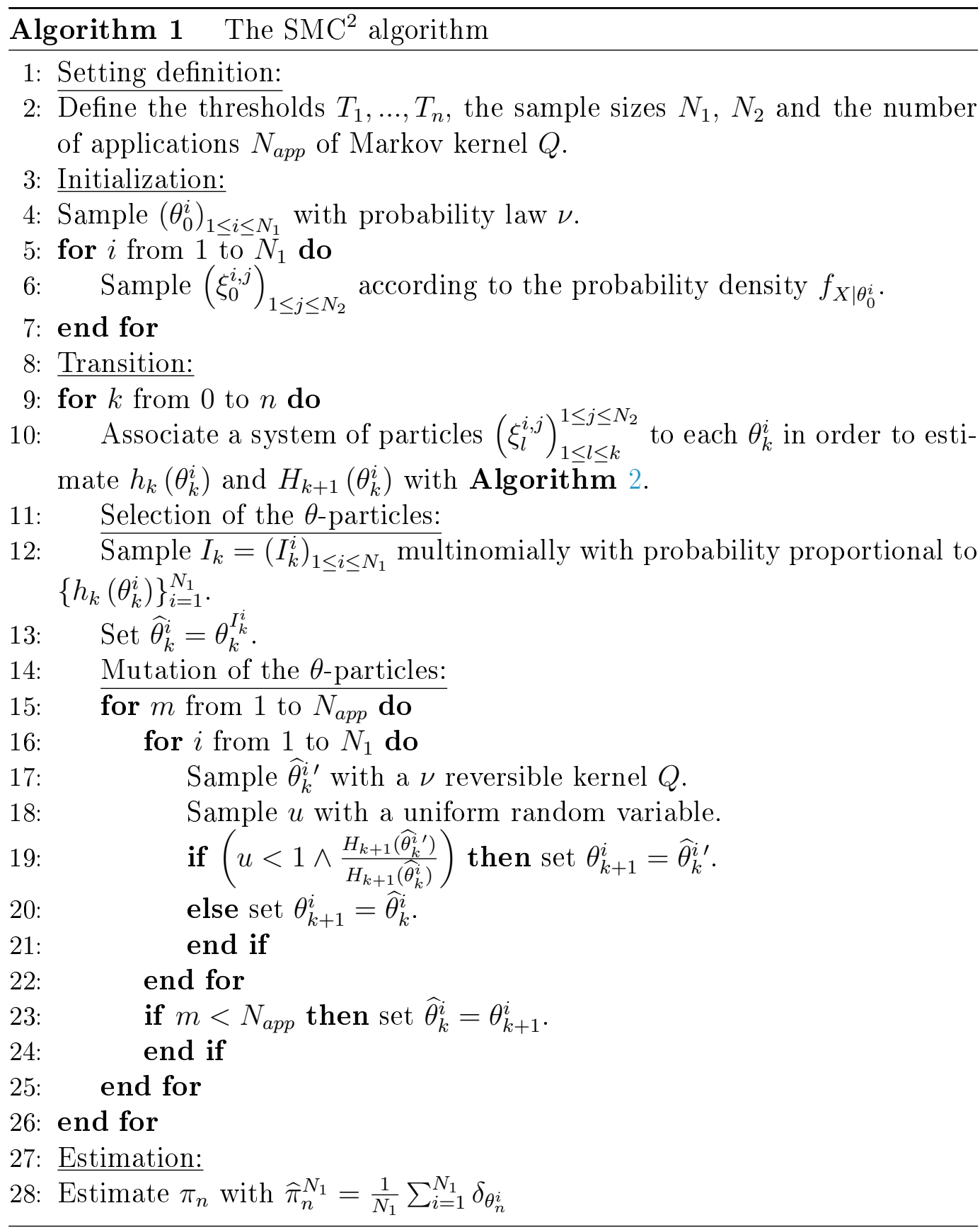

\section{Estimation of collision probability between orbiting objects}

The $\mathrm{SMC}^{2}$ algorithm has been applied on the debris satellite collision test case in order to estimate $\pi$, the conditional law of $\Theta$ given $\phi(X)<T$, with the following parameters: $N_{1}=1000, N_{2}=50, N_{a p p}=1, N_{a p p 2}=1$. The intermediate thresholds $T_{i}$ on the output distance are expressed in meters with $\{200,100,66,50,40,33,28,25,22,20\}$. The estimators of the different marginals of $\pi$, obtained with the $\mathrm{SMC}^{2}$ algorithm, are given in Figure 1, where the first marginal is related to the first parameter and so on. 


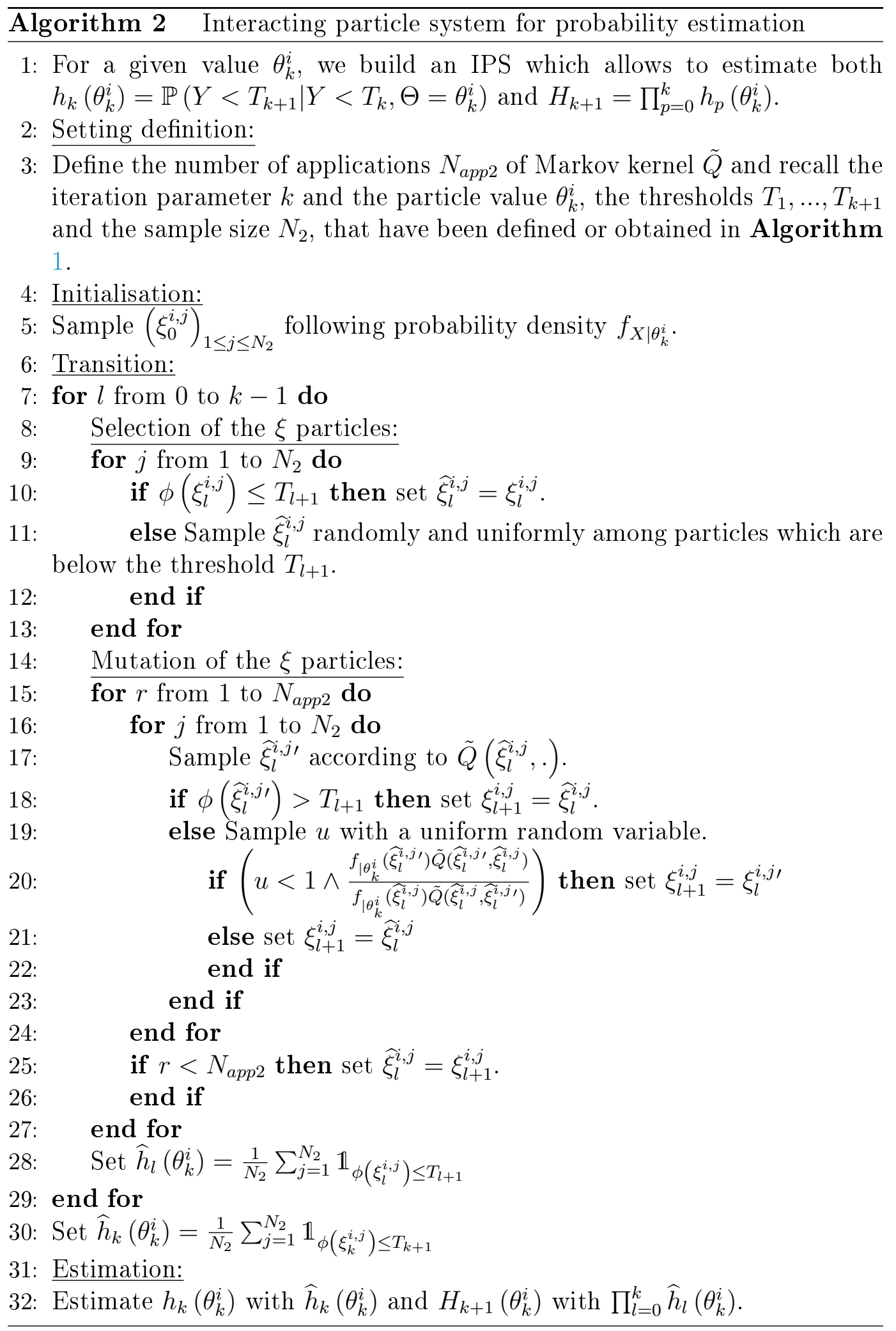

The estimated probabilities $\widehat{\mathbb{P}}(Y<T \mid \Theta=\theta)$, when $\Theta$ follows $\nu$ and $\pi$ are represented in Figure 2. The mean probability $\widehat{\mathbb{P}}(Y<T \mid \Theta=\theta)$ when $\Theta$ follows $\nu$ is estimated to $3.910^{-4}$. When $\theta=\sum_{i=1}^{N_{\theta}} \theta_{m}^{i} / N_{\theta}$, the probability 

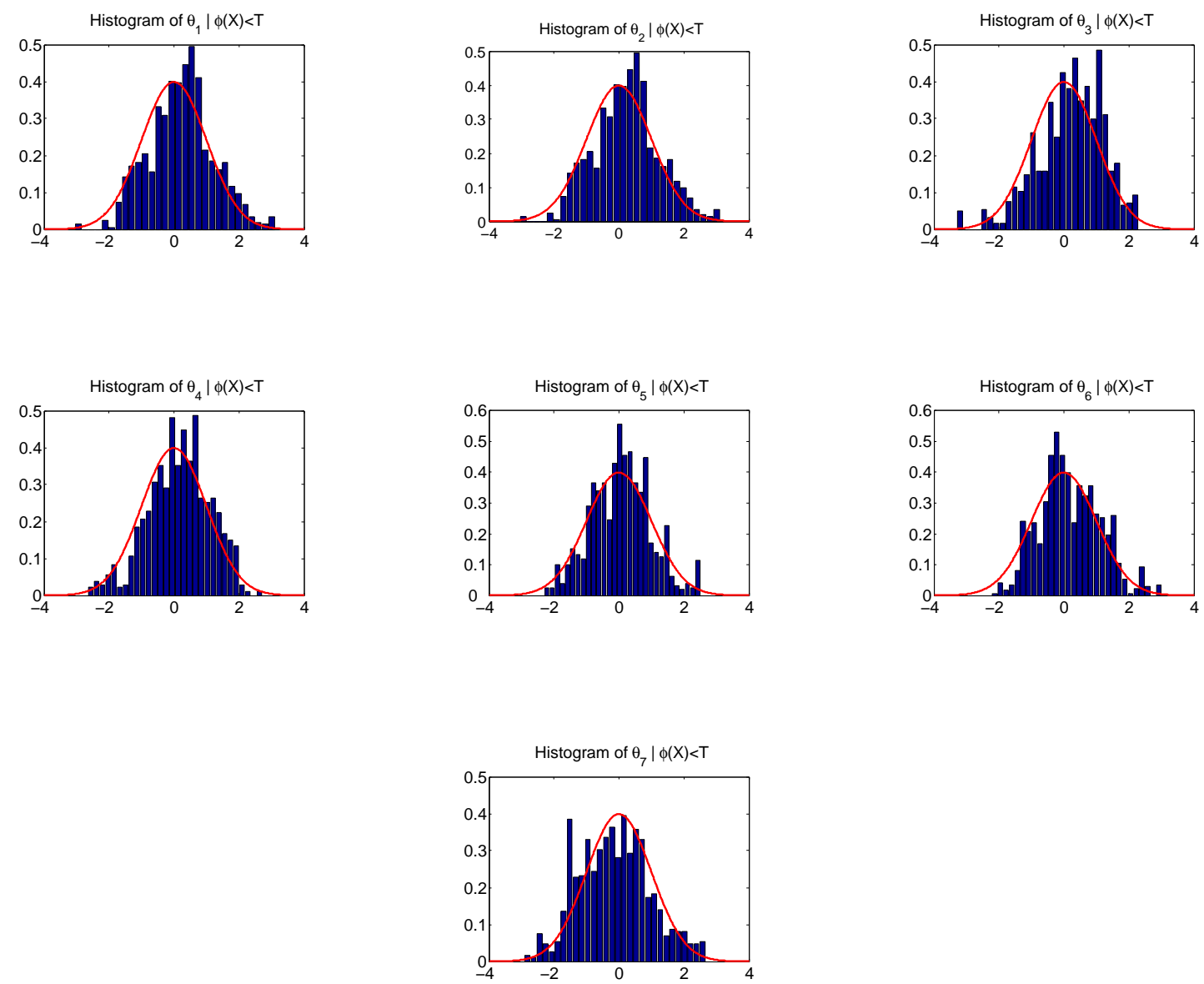

Fig. 1. Estimations of the marginals of $\pi$ using the $\mathrm{SMC}^{2}$ algorithm. The red curve corresponds to the standard normal density that is the initial marginal of the different parameters.

$\widehat{\mathbb{P}}\left(Y<20 \mid \Theta=\sum_{i=1}^{N_{\theta}} \theta_{m}^{i} / N_{\theta}\right)$ is equal to 0.034 .

The question is how to analyze the estimated density of $\pi$ for the tuning of $\Theta$. A possible approach is to consider the Kullback-Leibler distance between the estimated marginal density of $\pi$ for the parameter $\Theta_{i}$ and the initial marginal density of $\nu$ for parameter $\Theta_{i}$. If the Kullback-Leibler distance is significant for $\Theta_{i}$, then one can assume that $\Theta_{i}$ has to be finely tuned and conversely. In that case, a misestimation of $\Theta_{i}$ will indeed tend to increase the failure probability. Table 1 summaries the different Kullback-Leibler distance obtained for the different components of $\Theta$. The first error component $\Theta_{1}$ of the position vector seems to be the most influent parameter on $\mathbb{P}(\phi(X)<T)$. On the contrary, the second error component of position and speed vector, that are $\Theta_{2}$ and $\Theta_{5}$ require a lower accuracy since the considered values for these parameters 

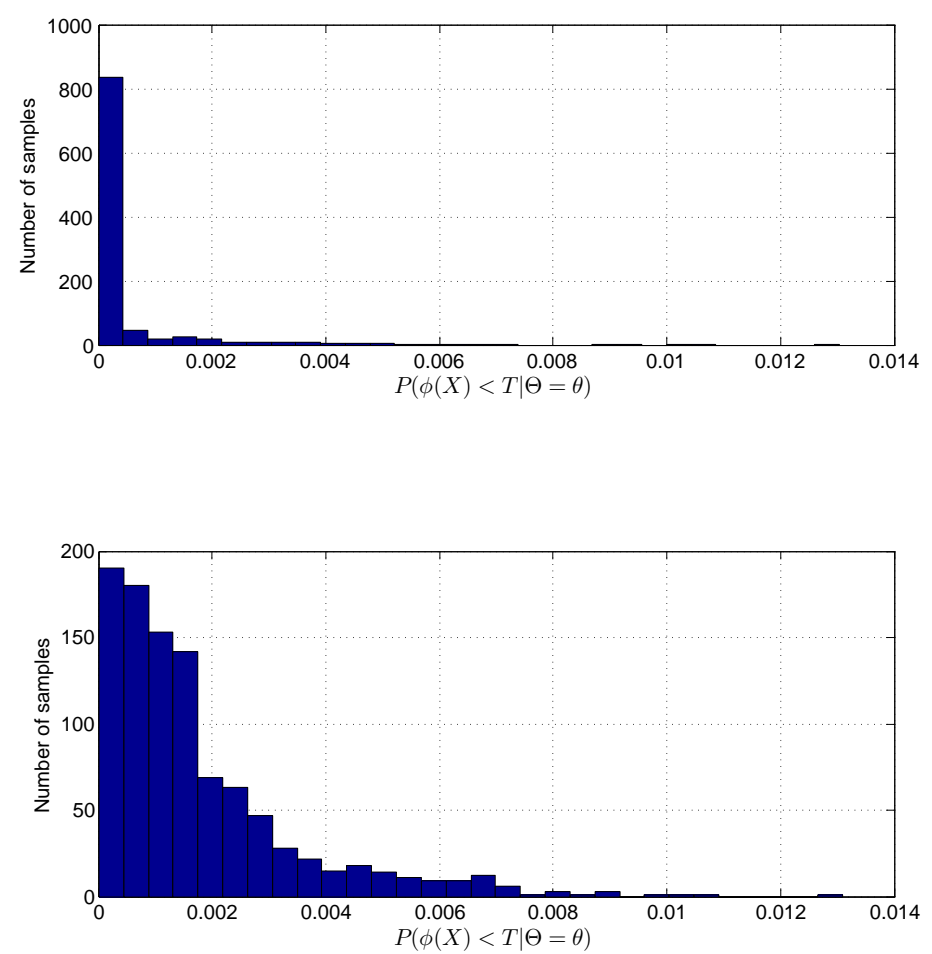

Fig. 2. Estimates of $\mathbb{P}(Y<T \mid \theta)$ with $\Theta$ following $\nu$ and $\pi$.

lead the maximum of the collision probability; an error on these parameters will thus tend to decrease the failure probability. In the same way, the density parameter $\Theta_{7}$ of the drag coefficient does not require also a too fine tuning in the proposed example.

It may be also interesting in practice to transform the six first components of $\Theta$ into usual orbital parameters (the semi-major axis $a$, eccentricity $e$, inclination $i$, argument of perigee $\omega$, longitude of the ascending node $\Omega$, the mean anomaly $m$ ) and then to evaluate $\pi$ in that case. The estimation of the marginals of $\pi$ for the different orbital parameters is proposed in Figure 3. The corresponding Kullback-Leibler analysis is given in Table 2. The mean anomaly is on this use case the orbital parameter that has to be most finely tuned. There is indeed a higher chance that the collision probability increases if the mean anomaly is not correctly set.

\section{Conclusion}

In this chapter, we have proposed an original methodology to analyze the influence of parameter model that are set for the sake of simplicity, on a rare 


\begin{tabular}{|c|c|}
\hline Component of $\Theta$ & Kullback-Leibler distance with the marginal of $\pi$ \\
\hline$\Theta_{1}$ & 0.46 \\
\hline$\Theta_{2}$ & 0.13 \\
\hline$\Theta_{3}$ & 0.30 \\
\hline$\Theta_{4}$ & 0.24 \\
\hline$\Theta_{5}$ & 0.11 \\
\hline$\Theta_{6}$ & 0.25 \\
\hline$\Theta_{7}$ & 0.10 \\
\hline
\end{tabular}

Table 1

Kullback-Leibler distance between marginal density $\pi$ and $\nu$ for parameters $\Theta_{i}$.

failure probability. The proposed $\mathrm{SMC}^{2}$ algorithm has been described in the case of a general problem where the model is a black-box system with random inputs. This algorithm has been applied with success for the analysis of collision probability between space debris and satellite. The set model parameters influence strongly the value of the collision probability and their value has to be carefully investigated to avoid collision probability underestimation.

The complete interpretation of target law $\pi$ remains complicated and has to be continued. The analysis of the particles obtained by the $\mathrm{SMC}^{2}$ algorithm with Sobol indices [Sobol and Kuchereko, 1993] is a potential perspective to this work.

\section{Acknowledgments}

The work presented in this chapter is part of a CNES/ONERA PhD thesis. 

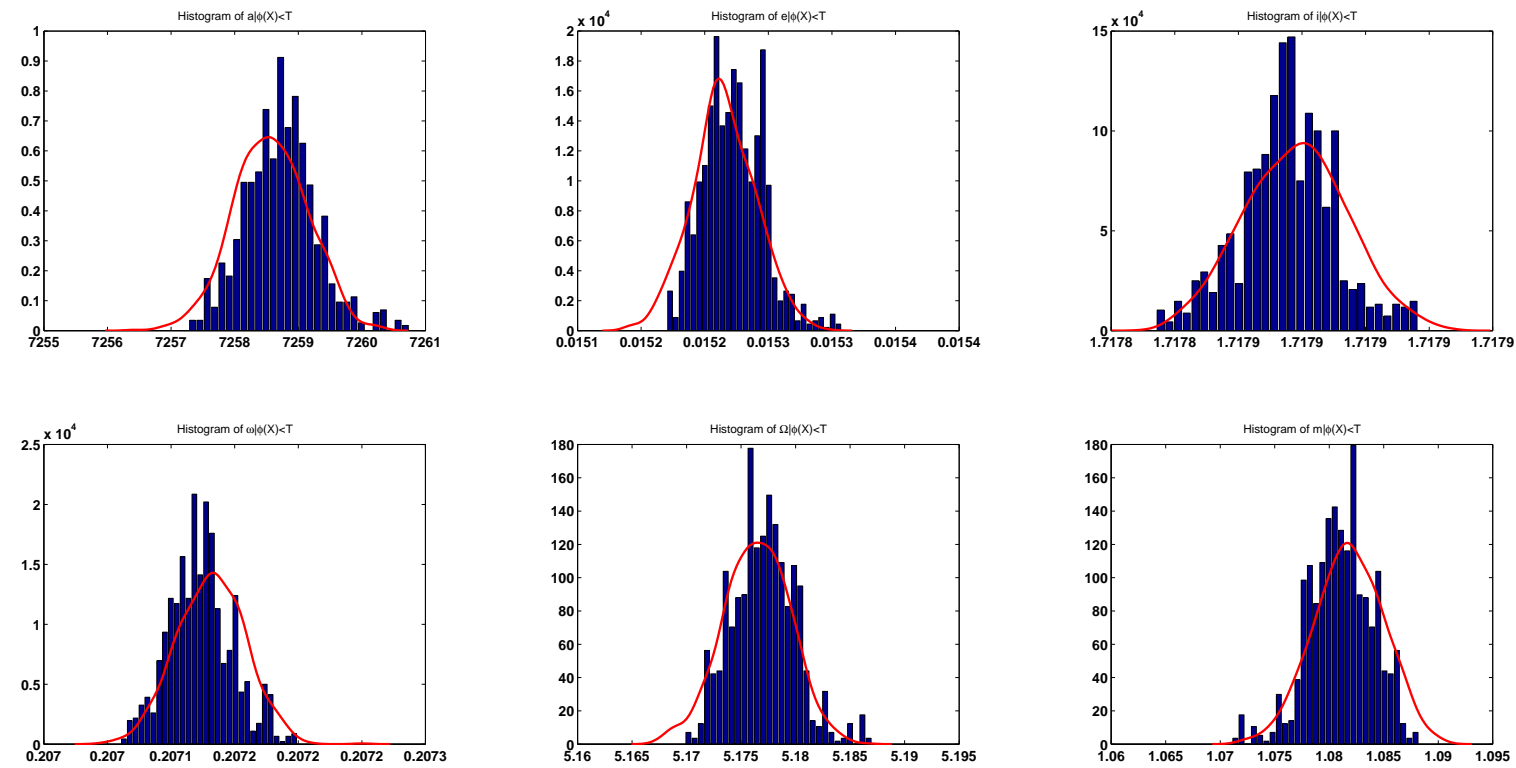

Fig. 3. Estimations of the marginals of $\pi$ using the $\mathrm{SMC}^{2}$ algorithm on the orbital parameters. The red curve corresponds to the initial density of the orbital parameters.

\section{References}

Bjerager, R. (1991). Methods for structural reliability computation, pages 89136. Springer Verlag, New York. 4

Botev, Z. I. and Kroese, D. P. (2012). Efficient Monte-Carlo simulation via the generalized splitting method. Statistics and Computing, 22(1):1-16. 4

Bucklew, J. A. (2004). Introduction to Rare Event Simulation. Springer. 4

Cérou, F., Del Moral, P., Furon, T., and Guyader, A. (2012). Sequential Monte Carlo for rare event estimation. Stat. Comput., 22(3):795-808. 4

Chopin, N., Jacob, P. E., and Papaspiliopoulos, O. (2013). SMC ${ }^{2}$ : an efficient algorithm for sequential analysis of state space models. Journal of the Royal Statistical Society: Series B (Statistical Methodology), 75(3):397-426. 2

Kelso, T. (2009). Analysis of the Iridium 33-Cosmos 2251 collision. In S. Ryan, T. M. E. D. B., editor, Proceedings of the Advanced Maui Optical and Space Surveillance Technologies Conference, page E3. Wailea, USA. 2

Lebrun, R. and Dutfoy, A. (2009a). A generalization of the Nataf transformation to distributions with elliptical copula. Probabilistic Engineering Mechanics, 24(2):172-178. 9

Lebrun, R. and Dutfoy, A. (2009b). An innovating analysis of the Nataf transformation from the copula viewpoint. Probabilistic Engineering Mechanics, $24(3): 312-320.9$

Miura, N. Z. (2009). Comparison and design of Simplified General Pertur- 


\begin{tabular}{|c|c|}
\hline Orbital parameters & Kullback-Leibler distance with the marginal of $\pi$ \\
\hline$a$ & 0.24 \\
\hline$e$ & 0.20 \\
\hline$i$ & 0.16 \\
\hline$\omega$ & 0.23 \\
\hline$\Omega$ & 0.15 \\
\hline$m$ & 0.34 \\
\hline
\end{tabular}

Table 2

Kullback-Leibler distance between marginal density $\pi$ and $\nu$ for the orbital parameters.

bation Models (SGP4) and code for NASA Johnson Space Center, Orbital Debris Program Office. PhD thesis, Faculty of California Polytechnic State University. 3

Moral, P. D., Hu, P., and Wu, L. (2012). On the Concentration Properties of Interacting Particle Processes, volume 3. Foundations and Trends in Machine Learning,Now Publishers. 5, 7

Nataf, A. (1962). Distribution des distributions dont les marges sont données. Comptes rendus de l'Académie des Sciences, 225:42-43. 9

Pei-Ling, L. and Kiureghian, A. D. (1991). Optimization algorithms for structural reliability. Structural Safety, 9(3):161-177. 9

Rosenblatt, M. (1952). Remarks on a multivariate transformation. Annals of Mathematical Statistics, 23:470-472. 9

Rubinstein, R. Y. and Kroese, D. P. (2004). The Cross-Entropy Method: a unified approach to combinatorial optimization, Monte Carlo Simulation and Machine Learning. Springer. 4

Sobol, I. and Kuchereko, S. (1993). Sensitivity estimates for non linear mathematical models. Mathematical Modelling and Computationnal Experiments, 1:407-414. 14

Sobol, I. M. (1994). A Primer for the Monte Carlo Method. CRC Press, Boca Raton, Fl. 4 
Tierney, L. (1994). Markov chains for exploring posterior distributions. Annals of Statistics, 22:1701-1762. 8, 9

Zhang, P. (1996). Nonparametric importance sampling. Journal of the American Statistical Association, 91(434):1245-1253. 4 